\title{
A large magnetic storage ring for Bose-Einstein condensates
}

\author{
A. S. Arnold, C. S. Garvie, and E. Riis \\ Dept. of Physics, University of Strathclyde, Glasgow G4 ONG, UK \\ www.photonics.phys.strath.ac.uk
}

(Dated: July 7, 2021)

\begin{abstract}
Cold atomic clouds and Bose-Einstein condensates have been stored in a $10 \mathrm{~cm}$ diameter verticallyoriented magnetic ring. An azimuthal magnetic field enables low-loss propagation of atomic clouds over a total distance of $2 \mathrm{~m}$, with a heating rate of less than $50 \mathrm{nK} / \mathrm{s}$. The vertical geometry was used to split an atomic cloud into two counter-rotating clouds which were recombined after one revolution. The system will be ideal for studying condensate collisions and ultimately Sagnac interferometry.
\end{abstract}

The field of atom optics [1] has seen a plethora of significant advances since the advent of laser cooling [2] and gaseous Bose-Einstein condensation (BEC) [3]. Analogs of mirrors, lenses, and beamsplitters for manipulating ultra-cold atoms now exist and are continually being improved.

A relatively recent addition to the atom-optical toolbox is the cold atom storage ring, where atoms are guided around a closed path. This is an interesting configuration for performing, for instance, ultra-sensitive Sagnac [4] atom interferometry. An electrostatic storage ring was first reported for molecules [5] in 2001. However, for many interferometry experiments atoms are more suitable candidates, as they can be easily laser cooled and/or prepared in the same quantum state.

The first atomic storage ring was formed with the magnetic quadrupole field created by two concentric current carrying loops [6]. Our storage ring [7] utilises the more symmetric quadrupole field of a four-loop geometry (Fig. (1). A third group has recently developed a storage 'stadium' 8] based on a magnetic waveguide. Our ring contains more than $5 \times 10^{8}$ atoms, has a lifetime of $50 \mathrm{~s}$ and an area of $72 \mathrm{~cm}^{2}$, each corresponding to more than an order of magnitude improvement with respect to Refs. 6, 8. The Sagnac effect [4 is linearly proportional to the area of an interferometer, and our ring's area compares favourably with that of a state-of-the-art thermal beam atom interferometer gyro $\left(A=0.22 \mathrm{~cm}^{2}[9]\right)$. The atoms can complete multiple revolutions in the ring, further increasing our effective area and thus the rotation sensitivity. In addition, our storage ring has the unique feature that we are able to form a BEC in a section of the ring [7] and observe its propagation around the ring.

We begin by considering the storage ring theoretically, before discussing our experimental setup. Comparisons will then be drawn between our experiment and theory.

All magnetic atom-optical elements make use of the Stern-Gerlach potential $U=\mu_{B} g_{F} m_{F} B$ experienced by an atom moving adiabatically in a magnetic field of magnitude $B$, where $\mu_{B}$ is the Bohr magneton, $m_{F}$ the atom's hyperfine magnetic quantum number, and $g_{F}$ is the Landé g-factor. To make a storage ring one must use atoms in weak-field-seeking magnetic states $\left(g_{F} m_{F}>0\right)$, which are attracted to minima of the magnetic field mag- nitude. Using the Biot-Savart law, one can express the cylindrically symmetric magnetic field from a single coil of radius $R, \mathbf{b}_{R}(r, z)$, in terms of elliptic integrals [10]. Our storage ring comprises four concentric circular coils (Fig. 1), with a toroidal quadrupole total magnetic field:

$$
\begin{aligned}
\mathbf{B}(r, z) & =\mathbf{b}_{R-\delta_{R}}\left(r, z-\delta_{z}\right)-\mathbf{b}_{R-\delta_{R}}\left(r, z+\delta_{z}\right) \\
& -\mathbf{b}_{R+\delta_{R}}\left(r, z-\delta_{z}\right)+\mathbf{b}_{R+\delta_{R}}\left(r, z+\delta_{z}\right) .
\end{aligned}
$$

In our experiment $R=5.0 \mathrm{~cm}, \delta_{R}=1.25 \mathrm{~cm}$, and $\delta_{z}=$ $1.35 \mathrm{~cm}$, leading to a ring of zero magnetic field at a radius $R_{0}=4.8 \mathrm{~cm}$ slightly smaller than the mean coil radius $R$. The axial wire (Fig. 1) adds a $1 / r$ azimuthal magnetic field to Eq. 1. which has little effect on the ring radius, but yields a storage ring with non-zero magnetic field.

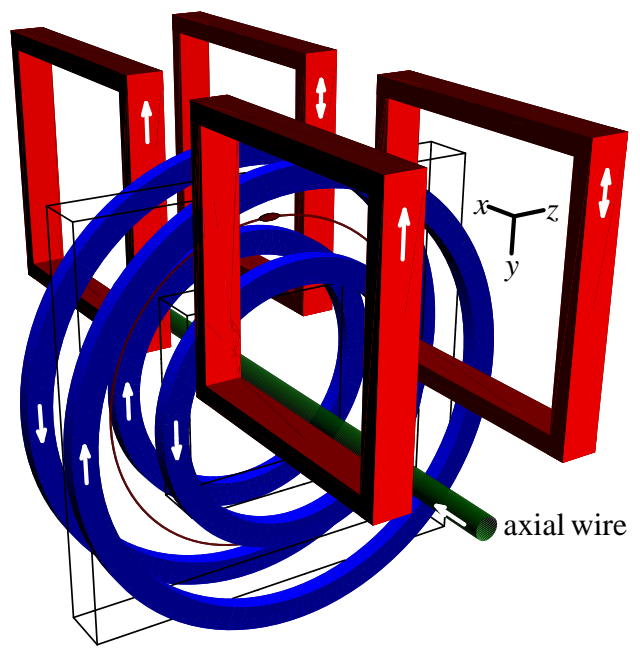

FIG. 1: (Color online) Four circular coils with average diameter $10 \mathrm{~cm}$ make up the storage ring (thin circle) to which the axial wire adds an adjustable azimuthal magnetic field. The square coils localise the atoms (small cigar) at the top of the ring in a magneto-optical (magnetic) trap if the left and right square coil pairs have the same (opposite) currents.

The theory of the storage ring for cold atoms is relatively simple, as the spatio-temporal evolution of each atom can be accurately described using the equations of motion arising from the Stern-Gerlach potential. Af- 
ter choosing suitable Gaussian initial position and velocity distributions it is possible to perform a Monte Carlo simulation to build up a spatio-temporal atomic density map.

By comparison with a $3 \mathrm{D}$ model, we have found that a $1 \mathrm{D}$ model is sufficient, i.e. the rigid pendulum equation:

$$
\theta^{\prime \prime}(t)=\left(g / R_{0}\right) \sin \theta
$$

where $\theta$ and $\omega=\frac{d \theta}{d t}$ are the angular position and velocity of an atom, $g$ is the acceleration due to gravity and $R_{0}$ is the radius of the storage ring. Note that Eq. 2 can be integrated analytically (using $\frac{d^{2} \theta}{d t^{2}}=\omega \frac{d \omega}{d \theta}$ ) given the initial angle $\left(\theta_{0}\right)$ and angular velocity $\left(\omega_{0}\right)$ of an atom, to determine the time-averaged relative probability of finding an atom at a given angle:

$$
P(\theta) \propto 1 / \omega=1 / \sqrt{\omega_{0}^{2}+2 g\left(\cos \theta_{0}-\cos \theta\right) / R_{0}} .
$$

In our vertically-oriented storage ring there is therefore a high time-averaged probability of finding atoms in the region where they travel the slowest - the top of the ring. The integral of Eq. 3] leads to an expression for $t(\theta)$ in terms of an elliptic integral, which can be inverted to find $\theta(t)$. There are two kinds of trajectories in the ring: if $\cos \left(\theta_{\max }\right)=\left|\frac{R_{0}}{2 g} \omega_{0}^{2}+\cos \theta_{0}\right|>1$ an atom will always rotate in the same direction, but if $\cos \left(\theta_{\max }\right) \leq 1$ (cf. Fig. 2(b)) the atom will reverse its direction around the ring at the turning points $\theta= \pm \theta_{\max }$.

The theoretical time-dependent angular distribution of atoms can be seen in Fig. 2. An atomic cloud centered in a parabolic potential can expand or shrink, but will maintain the same cloud shape. A circular atomic trajectory has a potential which is only parabolic to second order, and it is the higher order effects which cause an atomic cloud released from the top of the ring $(\theta=0)$ to break into two halves (Fig. 2(a)), leaving a near-zero probability at the top of the ring after around $300 \mathrm{~ms}$. These two halves return to the top of the ring after a further $300 \mathrm{~ms}$ and have a non-zero probability of remaining at the top of the ring for all subsequent times. The time for the two halves of the atomic cloud to 'recombine' has only a weak dependence on the initial atomic temperature. Gravity is the dominant effect for cold atoms, leading to a 'hot' velocity of $1.4 \mathrm{~m} \mathrm{~s}^{-1}$ at the bottom of the ring - a good location for future studies of high-energy $(20 \mathrm{mK})$ collisions between BECs [1].

Although the spread of initial thermal velocities quickly ensures that atoms reach the top of the ring at different times, in Fig. 2(a) we still see slowly decaying 'echos' of the originally localised spatial atomic cloud in the form of time-varying bimodal perturbations of the $\theta$ distribution. The angular width of these echos is approximately proportional to the initial cloud width, so the echos and their bimodal nature become more pronounced at higher temperatures.
In many of our ring experiments the atoms/BECs were not launched from $\overline{\theta_{0}}=0$ (Fig. 2(a)). Although the total number of atoms observed is similar to the $\overline{\theta_{0}} \neq 0$ case, the size of the returning $\overline{\theta_{0}} \neq 0$ cloud is much smaller and clearer due to focusing at the turning points of its motion (Fig. 2(b)).
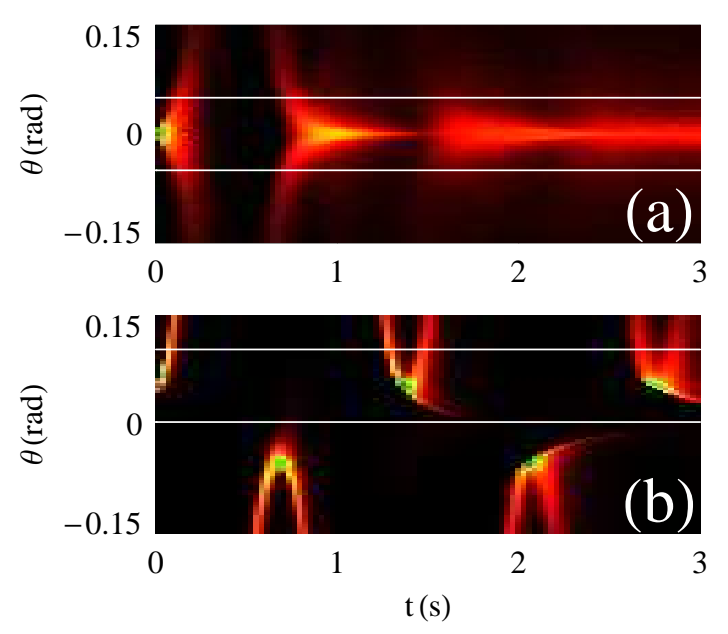

FIG. 2: (Color online) A Monte-Carlo simulation of atomic dynamics in the vertically-oriented storage ring for a $2 \mu \mathrm{K}$ initial cloud of atoms. Images (a) and (b) represent lossless atom dynamics for a cloud released at $\overline{\theta_{0}}=0$ and $\overline{\theta_{0}}=60 \mathrm{mrad}$ respectively. The white lines mark the extent of our $110 \mathrm{mrad}$ experimental viewing region.

We now turn to the experimental setup, for which many of the details are in Refs. 7, 12]. Our double magneto-optical trap (MOT) [13] collects $10^{9}{ }^{87} \mathrm{Rb}$ atoms in both our high and low pressure MOT chambers. The storage ring can be loaded directly from the low pressure MOT or Ioffe-Pritchard (IP [14]) trap without the need for any additional magnetic guiding or transfer, i.e. the MOT and IP trap are at the top of the ring. Releasing atoms at the top of a vertical ring ensures insensitivity to magnetic ring corrugations and a fast rotation frequency.

The advantage of our hybrid magnetic trap (Fig. 1) is that, using the same coils, it can be (i) a MOT, (ii) an IP magnetic trap (with trap frequencies $\nu_{r}=230 \mathrm{~Hz}$, $\nu_{z}=10 \mathrm{~Hz}$ ) or iii) a toroidal magnetic storage ring with radial gradient $230 \mathrm{G} / \mathrm{cm}$. In modes (i) and (ii) the magnetic field has an adjustable aspect ratio in the azimuthal direction. The four 2 turn $\times 500 \mathrm{~A}$ circular coils in Fig. 1 create a toroidal magnetic quadrupole field, confining the atoms to a ring. The four 3 turn $\times 500 \mathrm{~A}$ square 'pinch' coils are wired in pairs, and confine the atoms to a localised section of the ring for Ioffe-Pritchard trapping of the atoms. An azimuthal field can be added to the storage ring via an axial wire. The large coil sizes ensure good optical access, and low magnetic field noise. The absorption imaging (gravity) direction is the $x(y)$ axis.

After loading the low pressure MOT, the atomic cloud is optically pumped, loaded into the IP trap, magneti- 
cally compressed, and then evaporatively cooled to an adjustable temperature. The atoms can then be smoothly loaded into the storage ring in $20 \mathrm{~ms}$. We are able to form Bose-Einstein condensates containing $N_{0}=2 \times 10^{5}$ $\left|F=2, m_{F}=2\right\rangle$ atoms at the top of the storage ring [7] at a typical final RF evaporation frequency of $750 \mathrm{kHz}$.

In order to compare our experimental ring data (Fig. 3) with our theoretical model (Fig. 2), we have found it convenient to model the total number of atoms in the 'viewing window' of our absorption imaging system as a function of time. Our CCD camera has an area of $4.8 \times 6.4 \mathrm{~mm}^{2}$, with a magnification of $1.20(1)$. We have released cold atomic clouds with a variety of final evaporation temperatures into a storage ring with no azimuthal field. In accordance with theory, the atoms disappear after $300-400 \mathrm{~ms}$, reappear shortly afterwards, and are present at all subsequent times. However, there is a marked variation in the storage ring dynamics with atomic cloud release temperature. This can be explained in terms of non-adiabatic Majorana spin-flip transitions 15. The storage ring has a ring of zero magnetic field, and colder atoms pass closer to the magnetic field zero, and are selectively removed from the storage ring.
If we apply a constant magnetic field across the entire storage ring, perpendicular to the ring axis, it is possible to have only two places in the ring with zero magnetic field, however there will be a strong angular variation in the trapping potential. A novel feature of our storage ring is that the 'hole' in our quartz vacuum chamber (Fig. (1) allows us to use a wire along the axis of the storage ring. This means that we can generate an azimuthal magnetic bias field $B_{\theta}=0-10 \mathrm{G}$ around the ring which transforms the radial magnetic potential from a cone to a hyperbola, removing the ring of zero magnetic field and drastically reducing atomic loss.

This difference can be seen in the experiments of Fig. 3(a) and (b), where $2 \mu \mathrm{K}$ atomic clouds are released into a ring without and with an azimuthal field, respectively. A quantitative contrast of the number of visible atoms in each case is shown in Fig. 4 as well as a comparison to the Monte Carlo theory of Fig. 2 The difference the azimuthal field makes is even greater with condensates: without it BECs vanish before completing one revolution, but with an azimuthal field multiple revolutions of a condensate are possible with low loss (Fig. [3(c)).

\begin{tabular}{|c|c|c|}
\hline (a) & $=$ & 1 - \\
\hline 100 & 100 & 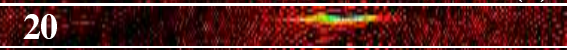 \\
\hline 200 & 200 & 40 \\
\hline 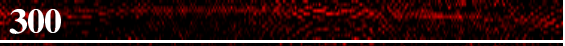 & 300 & 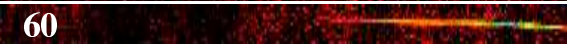 \\
\hline 400 & 400 & $80-28=$ \\
\hline 500 & 500 & 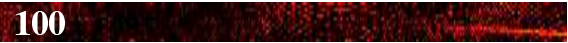 \\
\hline 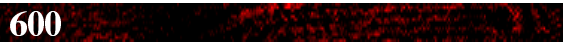 & 600 & $200-1200$ \\
\hline (1) & 700 & 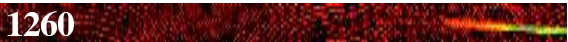 \\
\hline (2) & 800 & 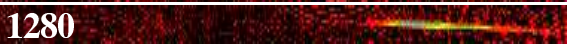 \\
\hline 60 & 900 & 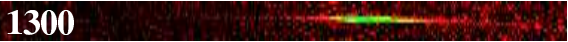 \\
\hline 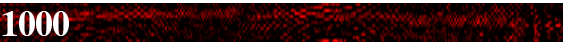 & 1000 & S \\
\hline 1100 & 1100 & 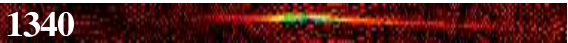 \\
\hline 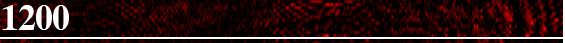 & 1200 & 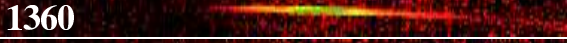 \\
\hline 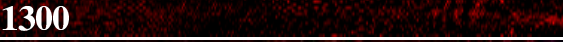 & 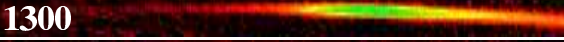 & W \\
\hline r n & 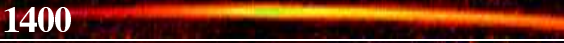 & 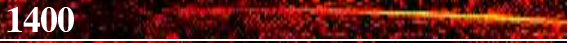 \\
\hline 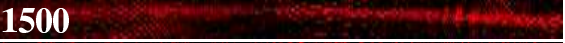 & 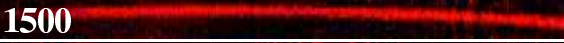 & 1420 \\
\hline 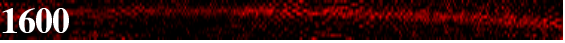 & 1600 & 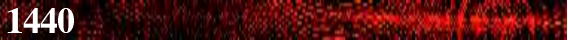 \\
\hline$x+5$ & 1700 & $1500-2500$ \\
\hline 1800 & 1800 & 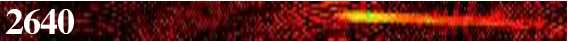 \\
\hline 1900 & 1900 & 2660 \\
\hline 2000 & 2000 & 2680 \\
\hline
\end{tabular}

FIG. 3: (Color online) Experimental storage ring dynamics for $2 \mu \mathrm{K}$ atoms with (a) $B_{\theta}=0 \mathrm{G}$ and (b) $B_{\theta}=10 \mathrm{G}$. In (c) multiple revolutions of a BEC in a ring with $B_{\theta}=10 \mathrm{G}$ are seen. Numbers denote time in ms, absorption images are $0.4 \times 5.4 \mathrm{~mm}^{2}$.

Note that when we performed experiments with an azimuthal field, the atoms/BECs were not launched from $\overline{\theta_{0}}=0$. If atoms are launched from $\overline{\theta_{0}}=0$ (Fig. 2(a)), then although the total number of atoms observed (Fig. (4) is similar to the $\overline{\theta_{0}} \neq 0$ case, the size of the returning $\overline{\theta_{0}} \neq 0$ cloud is much smaller and clearer due to focusing in the ring (Figs. 2(b), 3 $3(b, c)$ ).

We do not expect to see phase-fluctuations [16] in our condensate before or after propagation in the ring. These effects have been studied in highly elongated conden- 


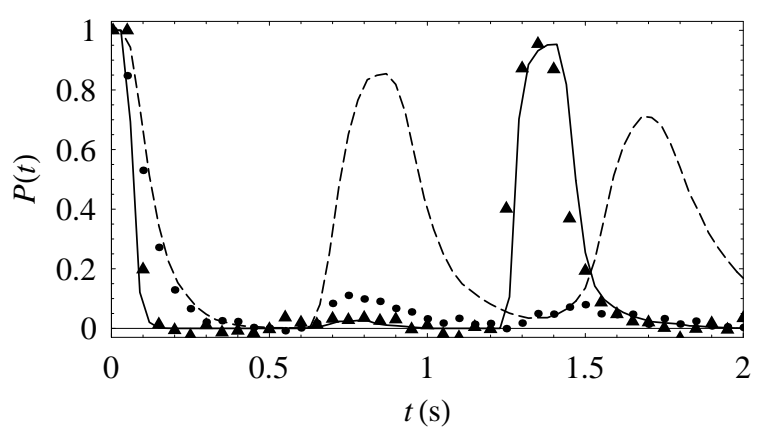

FIG. 4: The relative atomic population, $P(t)$, viewed by the $\mathrm{CCD}$ camera as a function of time for $2 \mu \mathrm{K}$ atoms. Experimental data with $B_{\theta}=0 \mathrm{G}$ and $B_{\theta}=10 \mathrm{G}$ are shown with circles and triangles respectively. The relative Monte Carlo atomic population in the viewing region of Fig. 2] a) (dashed curve) and Fig. 2(b) (solid curve) are shown for comparison. Good quantitative agreement can be seen between the $B_{\theta}=10 \mathrm{G}$ data and the Monte Carlo simulation of an atomic cloud released from $\theta_{0}=60 \mathrm{mrad}$. The $B_{\theta}=0 \mathrm{G}$ data only fits the expected dashed theoretical curve initially due to large Majorana losses.

sates in which the BEC coherence length is less that the length of the condensate. We form the condensate in only a moderately elongated trap, and the azimuthal expansion process is rapid enough that the (density-dependent) phase fluctuations do not have time to develop.

Sagnac interferometry will be performed by locating the condensate at the exact top of the ring, and incoherently splitting the sample by simply releasing it (Fig. 5 ). We are currently looking for interference fringes after a single revolution. Coherent splitting of the BEC will be achieved using Bragg scattering [17] to send BEC wavepackets in both directions around the ring. Note that ideally the phase sensitivity of a BEC interferometer scales like $\delta \phi_{B E C} \propto N^{-1}$ where $N$ is the number of atoms (cf. $\delta \phi_{T} \propto N^{-1 / 2}$ for thermal atoms), however the increased sensitivity is only possible if the BECs are prepared in number states 18.

\section{0}

1100

FIG. 5: A BEC at the top of the ring was split into two counter-rotating clouds by the faster-than-harmonic circular potential. These split atomic clouds can be seen recombining here after one revolution in the ring. Times are in ms, each image is $150 \times 3400 \mu \mathrm{m}^{2}$ and is taken after a $3 \mathrm{~ms}$ ballistic expansion.

In conclusion, we have demonstrated a storage ring for cold ${ }^{87} \mathrm{Rb}$ atomic clouds and BECs with an area of $7200 \mathrm{~mm}^{2}$. An azimuthal bias field around the ring enables low-loss BEC propagation with heating of less than $50 \mathrm{nK} / \mathrm{s}$. Our goal is a highly sensitive Sagnac atom interferometer, in which we are aided by our unprecedented ring area. Rotation sensitivity for a single revolution of $\Delta \Omega=\hbar /\left(8 m \pi R_{0}^{2} \sqrt{N}\right)=310^{-11} \mathrm{rad} / \mathrm{s}$ is feasible.

If one cannot create an azimuthal magnetic field, it is still possible to prevent spin-flip losses by using an adjustable-radius time-orbiting ring trap (TORT) 19]. We recently learnt that a team in Berkeley has created the first TORT for BECs [20], with ring area $5 \mathrm{~mm}^{2}$. Large BECs were also formed in an optically-plugged ring trap with ring area $\ll 1 \mathrm{~mm}^{2}[21]$.

We are grateful for helpful discussions with K. Burnett and E. Hinds. This work was supported by the UK EPSRC and the University of Strathclyde.

[1] C. S. Adams and E. Riis, Prog. Quant. Electr. 21, 1 (1997); E. A. Hinds and I. G. Hughes, J. Phys. D 32 , R119 (1999).

[2] S. Chu, L. Hollberg, J. E. Bjorkholm, A. Cable, and A. Ashkin, Phys. Rev. Lett. 55, 48 (1985); E. L. Raab, M. Prentiss, A. Cable, S. Chu, and D. E. Pritchard, Phys. Rev. Lett. 59, 2631 (1987).

[3] M. H. Anderson et al., Science 269, 198 (1995); K. B. Davis et al., Phys. Rev. Lett. 75, 3969 (1995); C. C. Bradley, C. A. Sackett, J. J. Tollett, and R. G. Hulet, Phys. Rev. Lett. 79, 1170(E) (1997).

[4] M.G. Sagnac, C.R. Acad. Sci. 157, 708 (1913).

[5] F. M. H. Crompvoets et al., Nature 411, 174 (2001).

[6] J. A. Sauer, M. D. Barrett, and M. S. Chapman, Phys. Rev. Lett. 87, 270401 (2001).

[7] C. S. Garvie, E. Riis and A. S. Arnold, Laser Spectroscopy XVI, P. Hannaford et al. eds., p178 (World Scientific, Singapore, 2004); see also www.photonics.phys.strath.ac.uk.

[8] S. Wu, W. Rooijakkers, P. Striehl, and M. Prentiss, Phys. Rev. A 70, 013409 (2004).

[9] T. L. Gustavson, P. Bouyer and M. A. Kasevich, Phys. Rev. Lett. 78, 2046 (1997).

[10] R. H. Good, Eur. J. Phys. 22, 119 (2001).

[11] N. R. Thomas, N. Kjærgaard, P. S. Julienne and A. C. Wilson, Phys. Rev. Lett. 93, 173201 (2004); Ch. Buggle, J. Léonard, W. von Klitzing and J. T. M. Walraven, Phys. Rev. Lett. 93, 173202 (2004).

[12] A. S. Arnold and E. Riis, J. Mod. Opt. 49, 959-964 (2002).

[13] K. Gibble, S. Chang and R. Legere, Phys. Rev. Lett. 75, 2666 (1995); C. J. Myatt et al., Opt. Lett. 21, 290 (1996).

[14] D.E. Pritchard, Phys. Rev. Lett. 51, 1336 (1983).

[15] W. Petrich, M. H. Anderson, J. R. Ensher, and E. A. Cornell, Phys. Rev. Lett. 74, 3352 (1995).

[16] D.S. Petrov, G.V. Shlyapnikov and J.T.M. Walraven, Phys. Rev. Lett. 87, 050404 (2001); S. Dettmer et al., ibid. 87, 160406 (2001); D. Hellweg et al., ibid. 91, 010406 (2003); S. Richard et al., ibid. 91, 010405 (2003).

[17] J. Stenger et al., Phys. Rev. Lett. 82, 4569 (1999); M. Kozuma et al., Phys. Rev. Lett. 82, 871 (1999).

[18] J. Jacobson, G. Bjork and Y. Yamamoto, Appl. Phys. B 60, 187 (1995).

[19] A.S. Arnold, J. Phys. B 37, L29 (2004).

[20] S. Gupta et al., Phys. Rev. Lett. 95, 143201 (2005).

[21] D. S. Naik, S. R. Muniz and C. Raman, Phys. Rev. A 72, 051606(R) (2005). 